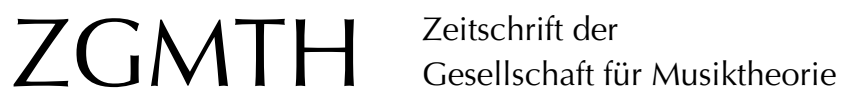

Sönksen, Sören (2013): Die Idee des stummen Fundamentes bei Rameau, Kirnberger und Sechter. ZGMTH 10/2, 373-387. https://doi.org/10.31751/730

\section{() 2013 Sören Sönksen}

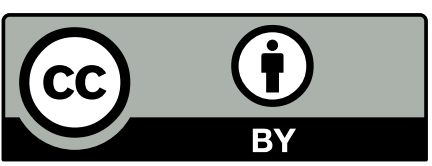

Dieser Text erscheint im Open Access und ist lizenziert unter einer Creative Commons Namensnennung 4.0 International Lizenz.

This is an open access article licensed under a

Creative Commons Attribution 4.0 International License.

veröffentlicht / first published: 17/10/2014

zuletzt geändert / last updated: 19/02/2016 


\title{
Die Idee des stummen Fundamentes bei Rameau, Kirnberger und Sechter
}

\author{
Sören Sönksen
}

ABSTRACT: Die Integration stummer, also theoretisch angenommener, aber nicht erklingender Akkordfundamente in die harmonische Analyse wird in diesem Aufsatz im Rahmen ihrer Entstehung bei drei einflussreichen Fundamentalbass-Theoretikern nachvollzogen. Dabei ist zu beobachten, wie sich die von Rameau für bestimmte Akkordfortschreitungen aufgestellten Erklärungshypothesen bei Kirnberger und Sechter zu Lehrsätzen und Regeln verfestigen, obwohl letztere Autoren die Methode einer physikalischen Fundierung nicht teilen. Rameaus Ansätze werden so einer Systematisierung unterzogen, die jedoch nicht widerspruchsfrei bleibt.

Im folgenden Beitrag soll ein Aspekt theoretischer Beschäftigung mit Musik untersucht werden, der von seiner Entstehung an bis heute kontrovers diskutiert wurde. Die Rede ist von der Praxis, bestimmte Zusammenklänge durch nicht erklingende Akkordgrundtöne zu erklären oder herzuleiten. Bereits 1776 weist Friedrich Wilhelm Marpurg, erstaunlicherweise in der Absicht, den `Grundbaß Rameaus gegen den >Interpolirbaß Kirnbergers abzugrenzen, auf ein zentrales Problem des Verfahrens hin:

Der reine Grundbaß kann nemlich nicht mehr oder weniger enthalten, als in den ausgearbeiteten Stimmen enthalten ist, und ist nur auf eine einzige Art möglich. Es ist damit wie mit dem Inhalt eines Buchs beschaffen, welcher so wenig ein Capitel angeben muß, das nicht darinnen befindlich ist, als ein darinnen befindliches auslassen. ${ }^{1}$

Was also bewog die Theoretiker dazu, ein intendiert bloß dokumentierendes Analyseinstrument buchstäblich in seinen Fundamenten zu verändern? Um die historischen Implikationen dieser Methode, also Kontinuitäten, Brüche, logische oder wissenschaftstheoretische Probleme, zu beleuchten, möchte ich mich dem Gegenstand anhand der Argumentationen dreier Theoretiker nähern, die einen entscheidenden Beitrag zur Genese der Idee des stummen Fundamentes geleistet haben. Dazu beziehe ich mich nachfolgend auf Jean-Philippe Rameaus Traité de I'harmonie, Johann Philipp Kirnbergers Die Kunst des reinen Satzes und Die wahren Grundsätze zum Gebrauch der Harmonie sowie auf Simon Sechters Grundsätze der musikalischen Komposition.

1 Marpurg 1776, 277. 


\section{Jean-Philippe Rameau}

Die Fundamentalbassstimme ( $`$ basse fondamentale`), Rameaus wohl berühmtestes analytisches Werkzeug, erweitert die bereits seit dem frühen 17. Jahrhundert bestehenden Beschreibungen des vom Basston eines Klanges abweichenden Grundtons ${ }^{2}$, indem sie nicht nur einzelne Fundamentaltöne anzeigt, sondern diese im Kontext einer Komposition wiedergibt, sodass die Fortschreitung der Grundtöne fokussiert wird. Dabei ergeben sich in Rameaus Deutungen neben einfachen Verhältnissen wie z.B. Quintrelationen auch ambivalente oder hinzugefügte Grundtöne, die nachfolgend als Ausgangspunkt der Idee des stummen Fundamentes dargestellt werden.

Ein die Rameau-Rezeption maßgeblich prägender Kritikpunkt besteht in der nachdrücklichen Betonung der musikalischen Vertikale durch den Theoretiker. Die bemängelte Reduktion auf starre harmonische Momentaufnahmen bzw. `Terzenstapek ergibt sich aus dem rationalistischen Grundprinzip Rameaus, seine Lehrsätze aus dem Einzelton (`son fondamentals) zu deduzieren. Für die von ihm hinzugefügten Fundamente ergibt sich hingegen ein gänzlich inverses Bild: Sie werden eingesetzt, um bestimmte Akkordfortschreitungen zu erläutern. Auch der vermeintlich paradigmatische Terzenaufbau der Akkorde zeigt sich dabei eher als Faustregel denn als Naturgesetz. Dies entspricht einem Verständnis der sbasse fondamentale` als Mittel zur Annäherung an horizontale Phänomene wie harmonische Progressionen oder die zuvor kontrapunktisch erklärte Dissonanzbehandlung. ${ }^{3}$

Für die Fortschreitung der sbasse fondamentale ‘ kommt Rameau zu dem Ergebnis, dass empirisch diejenigen Intervalle dominieren, die auch für die Entstehung eines konsonanten Akkordes aus den ersten Partialtönen des sson fondamental konstitutiv wirken, also Terz und Quinte. ${ }^{4}$ Durch diese Projektion des saccord parfaitı aus der Vertikalen in die Horizontale gelingt dem Autor die Anbindung des sson fondamental als grundlegendes sprincipe $\varsigma^{5}$ an die praktische, harmonisch bewegte Musik. Möglicherweise liegt in dieser Verknüpfung auch der Grund dafür, dass Rameau gerade die Bedeutung der Quinte für die Akkordbildung hervorhebt. ${ }^{6}$

Rameau gilt die natürliche sperfection` der Intervalle in der Reihenfolge ihrer Generierung gleichermaßen für die Harmonie und ihre Fortschreitung. Angesichts dieser Begründung verwundert es, dass er die Progression einer verminderten Quinte oder übermäßigen Quarte offenbar als unproblematisch erachtet oder diese Frage zumindest nicht anspricht. Im Sinne der Intervallhierarchie stellt das Streben der `basse fondamentale um eine Quinte abwärts die primäre harmonische Bewegung dar und lässt so die scadence imparfaite oder die `cadence rompuë، als Derivate der maßgeblichen `cadence parfaite` erscheinen. $^{7}$

\footnotetext{
2 Vgl. Dahlhaus 1989, 75.

3 Vgl. Christensen 1993, 26 und 54.

4 Rameau 1722, 50 f.

5 Vgl. den vollständigen Titel des Traités.

6 Rameau 1722, 12.

7 Christensen 1993, 113ff.
} 


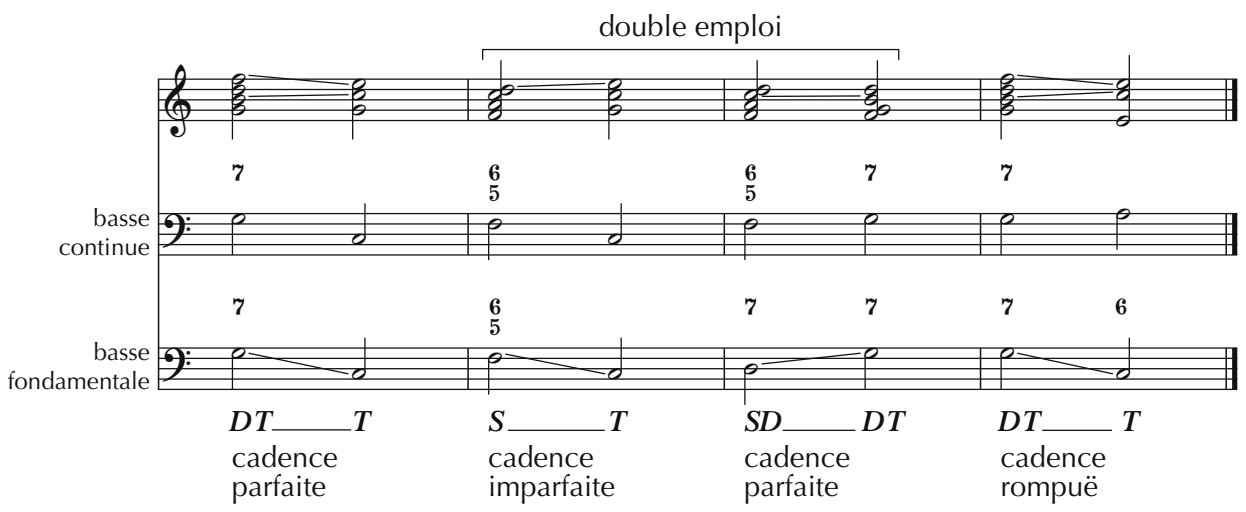

Beispiel 1: Grundtonambivalenz durch den ^double emploi` nach Jean-Philippe Rameau

Zu einem Fall von Grundtonambivalenz gelangt Rameau in Bezug auf den sdouble emploi<: Der Fundamentalbass der ssimples dominantes`(SD) und ssousdominantes` (S), die sich isoliert betrachtet lediglich durch Umkehrung voneinander unterscheiden, richtet sich nicht nach dem Terzenaufbau der Akkorde, sondern nach deren Fortschreitung. Je nach Auflösung der charakteristischen Dissonanz (mineure 7 bzw. majeure 6) unterscheidet er die Kadenzpaare (SD) $\rightarrow$ >dominante-tonique $(\mathrm{DT})$ sowie (S) $\rightarrow$ stonique $(\mathrm{T})$.

Ein besonderer Umgang mit dem Kadenzprinzip begegnet in Bezug auf die scadence rompuë،. Während Rameau einerseits versucht, den realen Basston als Substitut des Quinttons einer regulären stonique abzuleiten $^{8}$, verweist er andernorts gerade auf den steigenden Fundamentalbassschritt als Kadenzimitation. ${ }^{9}$ Dieses Paradoxon lässt sich nur auflösen, indem man den Erklärungsansatz der Substitution als `Analyse der Analyse` versteht, die lediglich die Verwandtschaft zur `cadence parfaite` herleitet und damit letztlich dem sprinciper geschuldet ist.

Nachfolgend soll auf einen für die Rezeption der Theorie der sbasse fondamentale besonders folgenreichen Aspekt hingewiesen werden: Um zu zeigen, dass diese Stimme sich vorrangig nach den Intervallen des saccord parfait richtet, eliminiert Rameau einige Sekundschritte durch die Interpolation eines weiteren Fundamentalbasstons ohne äquivalenten harmonischen Wechsel. Dies erscheint auf der Basis des >double emploi als ein sinnvoller Ausdruck der mit diesem Phänomen verbundenen Grundtonambivalenz. Daher überrascht es, dass Rameau (in Beispiel 2) den zusätzlichen Fundamentalbasston nicht unter, sondern vor den fraglichen Klang setzt (a).

Bei (b) wird der Sekundschritt G-A in der sbasse fondamentale ‘ durch ein zusätzliches E erweitert, bei der mit (a) vergleichbaren Stelle (c) hingegen nicht. Die abweichende Bezifferung der beiden Stellen (a) und (c) vermag dies nicht zu begründen, da Rameau in Takt 7.1 der sfalschen Auflösung der sdissonance mineure Trotz einen Septakkord über A analysiert. Derlei Beispiele für die unterschiedliche Deu-

8 Ebd., $116 f$.

9 Rameau 1722, 75. 


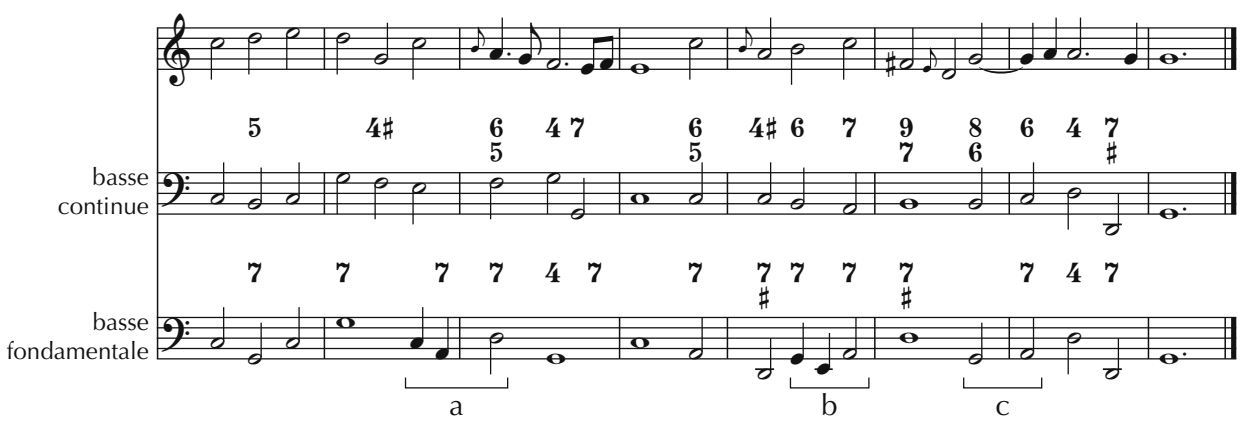

Beispiel 2: Jean-Philippe Rameau, Traité de I'harmonie réduite à ses principes naturels, Interpolation von Fundamentalbasstönen bei Sekundschritten der ‘basse fondamentale $\iota^{10}$

tung analoger Stellen bzw. den lediglich sporadischen Gebrauch des interpolierten Tones lassen an der Plausibilität des Verfahrens zweifeln.

Ein weiterer Bestandteil der Rameau'schen Theorie, der das Prinzip des Terzenaufbaus der Akkorde schwächt, kann im Modell der ısupposition` erkannt werden. Dessen Herleitung ist eng mit derjenigen des Septakkordes verwandt. Dort greift Rameau auf die Kombination von geschichteten Terzen zurück ${ }^{11}$, was in der Folge auf Nonen- und Undezimenakkorde übertragen wird. Um das Diktum des srenversement zu können, schließt der Autor den Basston solcher Fünfklänge aus der Harmoniestruktur aus. Der verbleibende Vierklang behält seine Umkehrbarkeit, wohingegen der reale Basston an seine Position gebunden ist. ${ }^{12}$

Das folgende Notenbeispiel zeigt, dass Rameau auch den Quartvorhalt auf der Antepänultima durch ssupposition erklärt (a). Bei dieser Verwendung werde der Akkord jedoch für gewöhnlich unvollständig gesetzt, um klangliche Härten zu vermeiden. ${ }^{13}$ Dies bildet ein Extrembeispiel für die vertikale Herleitung von satztechnischen Phänomenen. Dennoch sollte ebenfalls beachtet werden, dass Rameau durch die Erklärung der Quarte zur eigentlichen Septime ihre reguläre horizontale Bewegung als `dissonance mineure` begründet. Auf die Stimmführung der Septime über dem realen Basston eines vollständi-

10 Rameau 1722, 325.

11 Ebd., 30f.

12 Ebd., 74.

13 »Et finalement, que l'Accord de la Onziéme, chiffré par un 4. étant extrémement dur dans sa composition ordinaire, on en retranche presque toûjours les Sons moyens, en conservant seulement les deux principaux, qui sont le Fondamental \& sa Septiéme, \& quelquefois encore la Tierce mineure ou sa Quinte, ausquels on substituë un nouveau Son qui se met une Quinte au dessous du fondamental, \& et qui fait par consequent la Onziéme, \& non pas la Quarte avec la Septiéme du fondamental.« ) [»Und da schließlich der durch eine 4 bezifferte Undezimenakkord in vollständiger Setzung extrem hart klingt, lässt man dessen Mittelstimmen fast immer entfallen, sodass lediglich die zwei Hauptnoten Grundton und Septime sowie manchmal die kleine Terz oder die Quinte verbleiben, die durch einen neuen Ton ausgetauscht werden, der sich eine Quinte unter den Grundton legt und daher mit der Septime des Grundtones die Undezime und nicht die Quarte bildet.«] (Ebd., 76) 
gen Nonenakkordes, also der nach Rameau eigentlichen Quinte, kann diese Herleitung jedoch nicht übertragen werden. Ob der fragliche Akkordbestandteil also als Konsonanz behandelt werden kann, bleibt offen, da er bis auf den Orgelpunkt in Takt 4 (c) traditionell vorbereitet und aufgelöst wird (b):

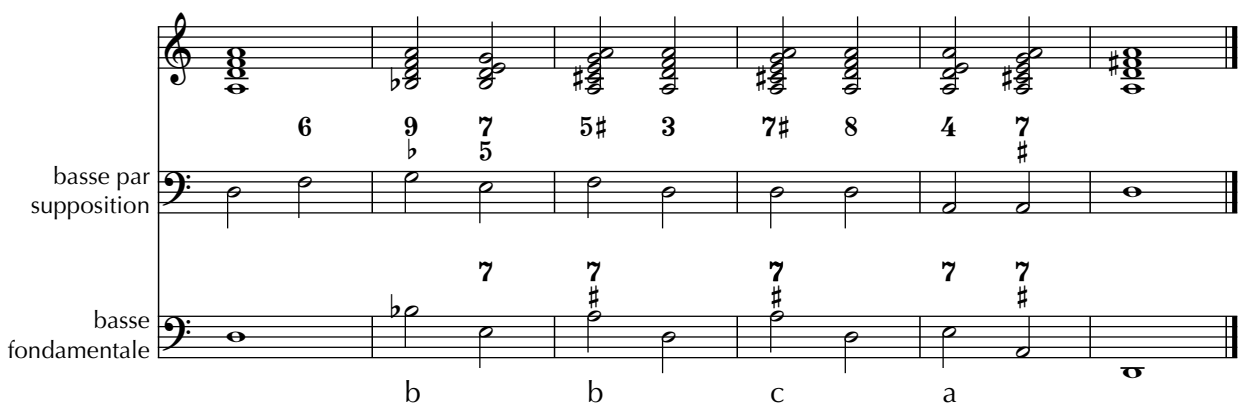

Beispiel 3: Jean-Philippe Rameau, Traité de l'harmonie réduite à ses principes naturels, Stimmführung von raccords par supposition ${ }^{14}$

Generell werden Vorhalte als sich über demselben Fundamentalbasston auflösende ınotes de goût ‘ von durch ssupposition ‘ entstehenden Akkordbildungen unterschieden, die durch ihre fundamentale Septimdissonanz einen Harmoniewechsel erfordern. Im analytischen Umgang jedoch zieht Rameau die Trennungslinie zwischen beiden Phänomenen nicht systematisch, sondern pragmatisch nach den jeweiligen Erfordernissen des Analysekontextes. ${ }^{15}$

Auf eine wiederum andere Weise wird schließlich der vollverminderte Septakkord erklärt, indem er durch Auswechslung des Septimtones auf einen kleinen Dur-Septakkord zurückgeführt wird. Hier liege also keine ssupposition`vor, sondern vielmehr eine Substitution:

Pour distinguer ce dernier accord, \& ses dérivez de celuy dont ils tirent leur origine, nous les appellerons, empruntez; en ce qu'ils empruntent leur perfection d'un Son qui n'y paroît point. ${ }^{16}$

Durch diese Begründung erhält Rameau die Umkehrbarkeit des vollverminderten Septakkordes und stattet inn, wenngleich ideell, mit einer reinen bzw. perfekten Quinte aus.

Wenn es sich bei den angeführten Beispielen auch um keine explizite Verkürzung der Akkorde handelt, so sollte daraus doch hervorgehen, dass die Bestimmung des ison fondamentak keinesfalls auf die Formel des tiefsten Tons einer Terzenschichtung zu bringen ist, sondern unterschiedliche Erklärungsstrategien Anwendung finden, die durch die

14 Rameau 1722, 75.

15 Vgl. Christensen 1993, 123-129. Das Verhältnis von ssupposition ‘ und Vorhaltsbildung in Rameaus späteren Werken wird in Martin 2012 besprochen.

16 Rameau 1722, 43. 
Orientierung an Akkordprogressionen den Terzenaufbau mitunter durchbrechen oder durch nicht erklingende Töne modifizieren.

Abschließend möchte ich auf ein späteres Analysebeispiel Rameaus aus dem Code de musique pratique ${ }^{17}$ hinweisen, aus dem ersichtlich wird, dass die beschriebenen Herleitungen nicht unbedingt auch Eingang in die tatsächliche Analyse finden. Dazu gehören der Fundamentalbass der >cadence rompuë، (a) sowie des vollverminderten Septakkordes (b) und das Fehlen von interpolierten Grundtönen (c).
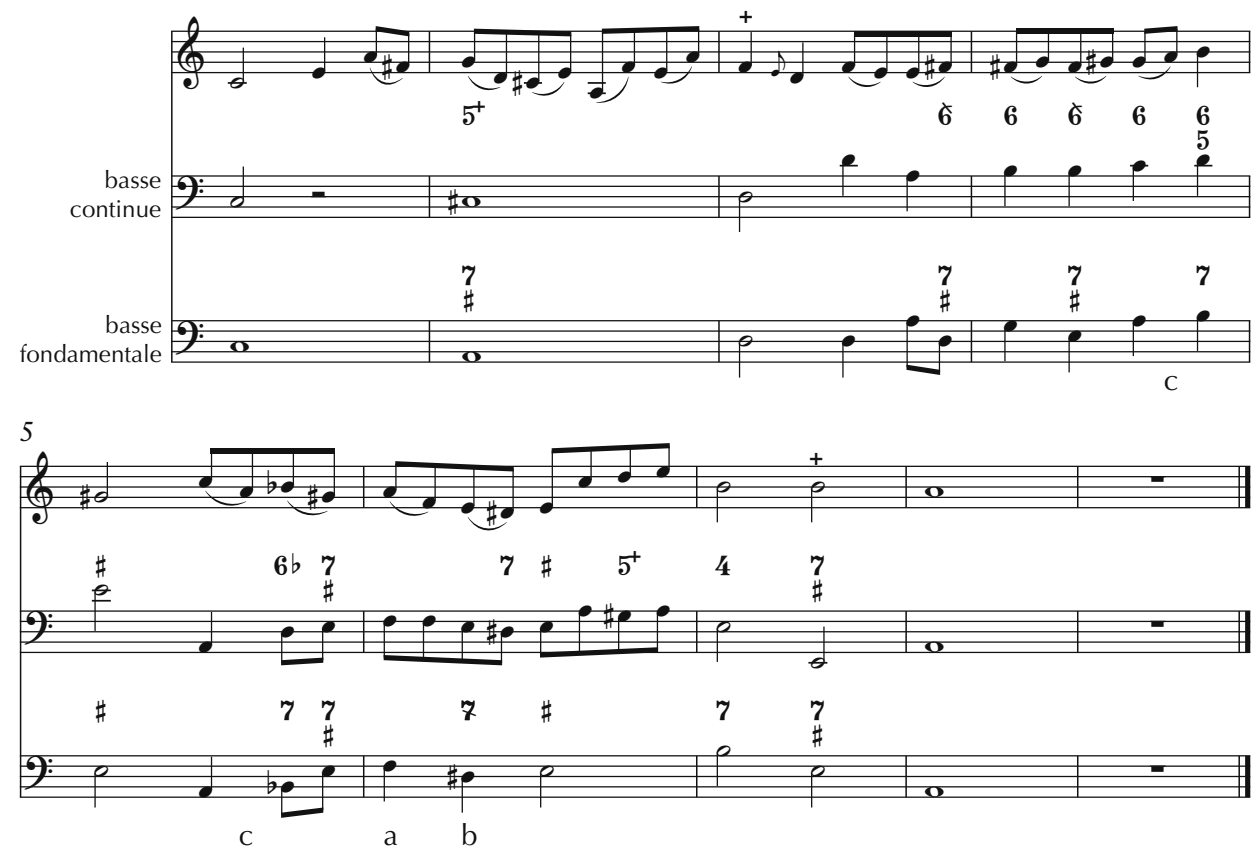

Beispiel 4: Jean-Philippe Rameau, Code de musique practique, Abweichungen von den besprochenen Deutungen ${ }^{18}$

\section{Johann Philipp Kirnberger}

Das Verhältnis Kirnbergers zur Theorie Rameaus gestaltet sich insofern problematisch, als

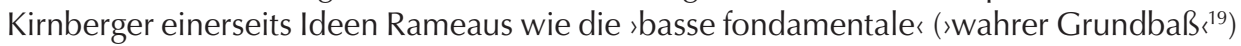

17 Rameau 1760.

18 Ebd., 27.

19 Im Gegensatz zur Herangehensweise Rameaus ist der `GrundbaßくKirnbergers von seiner Einführung an mit dem Anspruch auf Eindeutigkeit bzw. Wahrheit verknüpft: »Der Grund-Baß, der hier auf dem untersten Linien-System steht, enthält die wahren Grund-Töne zu den verschiedenen Accorden $[\ldots] \ll .(1771,54)$ 
adaptiert, andererseits jedoch dessen System als Ganzes ablehnt. ${ }^{20}$ An die Stelle eines rationalistischen Grundprinzips tritt die Orientierung an der Autorität großer (deutscher) Komponisten. ${ }^{21}$ Die Unterschiede zur Fundamentalbasslehre Rameaus sind zwar insgesamt überschaubar, betreffen jedoch in besonderem Maße diejenigen satztechnischen Phänomene, bei denen sich schon in der Darstellung Rameaus Schwierigkeiten in der Grundtonbestimmung erwiesen haben. Sie sind damit für die Entwicklung der Idee des stummen Fundamentes bedeutsam.

Grundlegend für das Verständnis der Lehre Kirnbergers ist die prominente Unterscheidung von szufälligen nung eine zentrale Rolle bei der Identifizierung der `Grundaccorde`.

Es erhellet deutlich [...], daß die auf diese Art entstandenen Dißonanzen fast allemal, wo sie vorkommen, könnten weggelassen werden, ohne daß dadurch irgend ein Fehler oder eine Zweydeutigkeit entstehet. Da also diese Vorhälte nicht nothwendig sind, so wollen wir sie zufällige Dißonanzen nennen. Ausser diesen Dißonanzen giebt es noch eine andere Art, die man nothwendige oder wesentliche nennen kann, weil sie nicht an der Stelle einer Consonanz gesetzt werden, der sie gleich wieder weichen, sondern eine Stelle für sich behaupten. ${ }^{22}$

Grundsätzlich werden hier harmoniefremde ızufällige` Dissonanzen von harmonieeigenen swesentlichen Septimtönen unterschieden. ${ }^{23}$ So einleuchtend diese Trennung erscheint, so schwierig erweist sie sich in Zweifelsfällen. Das genannte Unterscheidungsmerkmal des sofortigen Weichens wird dadurch unscharf, dass auch ızufällige Dissonanzen die Lizenz zur Auflösung über der folgenden Harmonie erhalten, «als ob sie wesentlich wären. « ${ }^{24}$ Auch die »Behauptung einer Stelle für sich« erscheint problematisch, wenn man sich vergegenwärtigt, dass die vier von Kirnberger/Schulz (s. Fußnote 20) genannten ıwesentlichen Septakkorde ${ }^{25}$ ebenfalls als ızufällige` Dissonanzen denkbar sind und auf diese Weise in ıVerkehrungen eines jeweils anderen ıwesentlichen Septakkordes münden, wie nachstehendes eigenes Beispiel verdeutlicht:

20 Vgl. Kirnberger/Schulz 1773, 4. Kirnbergers Schüler Johann Abraham Peter Schulz behauptete seine Autorschaft der Grundsätze nach Kirnbergers Tod. Eine Zusammenfassung der diesbezüglichen Kontroverse findet sich in Lester 1992, 240. Kirnbergers Haltung geht jedoch gleichfalls aus dem Vorbericht zu Friedrich Wilhelm Marpurgs Versuch über die musikalische Temperatur (1776) hervor: »Der Hr. Kirnberger besuchte mich öfters während den [sic] Abdruck seines Werks über den musikalischen Grundbaß, und erzählte mir, wie er die Rameauisten in die Enge treiben würde.«

21 Bezeichnend ist in dieser Hinsicht auch das konkrete Aufzeigen von Belegen oder Ausnahmen wie in Kirnberger 1781, 83: »In J. Seb. Bach sämmtlichen Compositionen habe ich bei einem einzigen Vorfall in einem vierstimmigen Satze auf dem Oberdominanten-Accord die große Terz verdoppelt gefunden $[. ..] \ll$.

22 Kirnberger 1771, 30.

23 Vgl. ebd., 27-31.

24 Kirnberger/Schulz 1773, 17.

25 Ebd., 5. 


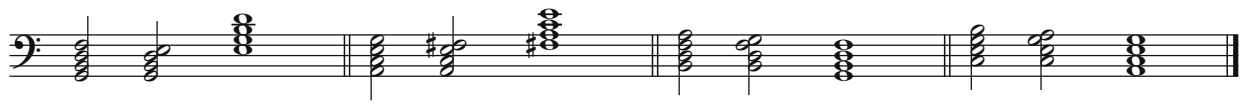

Beispiel 5: Identität der «wesentlichen`Septakkorde durch ızufällige` Vorhaltsbildungen

Zuletzt kann lediglich der Hinweis auf die ıZweydeutigkeit die Unterscheidung der beiden Dissonanztypen in Zweifelsfällen unterstützen: Kirnberger bemerkt zu der Folge C-G: »Auf dieser Stelle nun wird das Gehör des Kenners ungewiß, zu welchem Ton diese Harmonie b [G] gehöre. ${ }^{26}$ Um die Mehrdeutigkeit C-Dur: I-V versus G-Dur: IV-I zu klären, wird die Harmonie G um ihre lediglich im Tonvorrat der C-Dur-Skala zu findende kleine Septime ergänzt. Damit gehört zum Wesen der swesentlichen tonal verdeutlichende Rolle. Vor dem Hintergrund dieser Überlegungen erscheint die Exemplifizierung der vier swesentlichen Septakkordformen durch ihr charakteristisches Vorkommen innerhalb mehrstufiger Kadenzmodelle sogar notwendig:

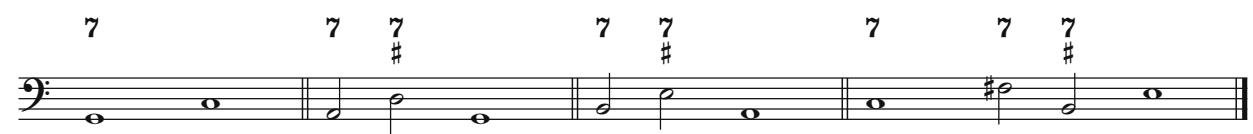

Beispiel 6: Johann Philipp Kirnberger / Johann Abraham Peter Schulz: Die wahren Grundsätze zum Gebrauch der Harmonie, Einbindung der vier swesentlichen، Septakkordformen in mehrstufige Kadenzmodelle ${ }^{27}$

Kirnberger/Schulz kombinieren die prototypische Verwendung einer Klangform mit den ordnenden Prinzipien der Umkehrbarkeit und der Quintfortschreitung des Fundamentes, wie sie bei Rameau als `renversement und ssimple dominante vorgebildet sind.

Es wird daraus verständlich, warum mehrmals auf die entscheidende Rolle des harmonischen Umfeldes einer zu bestimmenden Harmonie verwiesen wird: ${ }^{28}$ Der swahre Grundbaß< lässt sich nicht zweifelsfrei aus einem isolierten Akkord ableiten.

Anhand dieses Verständnisses der ıwesentlichen` Septakkorde überprüft Kirnberger abweichende oder mehrdeutige Verbindungen jeweils auf die Möglichkeit ihrer Integration in den beschriebenen kadenziellen Zusammenhang, ohne dies jedoch ausdrücklich als Methode zur Fundamentalbassbestimmung zu beschreiben. Um Ähnlichkeiten zu der prototypischen Verwendung der swesentlichen` Septakkorde hervorzuheben, deutet Kirnberger Akkordtöne einerseits als ızufälliges Dissonanzen und fügt den fraglichen Klängen andererseits funktional klärende Töne hinzu ${ }^{29}$, wie ich anhand einiger Beispiele demonstrieren werde.

26 Kirnberger 1771, 31.

27 Kirnberger/Schulz 1773, 6.

28 Z. B. ebd., 23 und 104.

29 Letztere nachfolgend entscheidende Praxis ist keine Erfindung Kirnbergers, sondern gehört zur damals alltäglichen Interpretation einer Bezifferung; vgl. Lester 1992, 57f. Von Rameau wird das unterschwellige Mithören möglicher Ergänzungstöne als ssous-entendre beschrieben. 
Der vollverminderte Septakkord etwa könne ausschließlich einen Halbton über seinen Basston in Terzenschichtung geführt werden ${ }^{30}$, wie z. B. in der Verbindung Moll: VII ${ }^{7}-$ I. Er wird als mit einer zzufälligen Septime versehener suneigentlicher Septimenakkord $^{31}$ bezeichnet. Auf Grund dieser funktionalen Eindeutigkeit nach dem Modell des ersten oben genannten Prototyps kann hier im Sinne Kirnbergers von einem ıwesentlichen`Septakkord gesprochen werden, dessen zusätzlich ızufällig` vorgehaltener Grundton erst über der folgenden Harmonie erscheint. Somit wird dieses Phänomen erklärt, ohne mit einer Verkürzung des Akkordes operieren zu müssen. Lediglich das Argument des unvorbereiteten Eintritts, den Kirnberger explizit erlaubt ${ }^{32}$, kann auf diese Weise nicht widerlegt werden.

Der verminderte Dreiklang hingegen habe »keine andere Fortschreitung, als vier Grade über sich zu treten. «33 Als konsonierender ${ }^{34}$, seigentlicher Akkord bildet er den Grundakkord für den dritten oben aufgeführten Prototyp der swesentlichen septakkorde. Bei seiner schrittweisen Progression als Sextakkord auf einer zweiten Stufe habe er allerdings »seinen Ursprung vom [Dominant-]Septimen-Accord. (35 $^{35}$ Damit wird implizit auf den eigentlichen `Grundbaß in Gestalt des Grundtons dieses Septakkordes und damit auf den suneigentlichen Charakter des real klingenden Akkordes verwiesen. Die Erklärung durch die verzögerte Auflösung einer ızufälligen` Dissonanz ist jedenfalls nur im Falle einer hinzugefügten Septime möglich. ${ }^{36}$ Beim verminderten Dreiklang in der Fortschreitung Dur: VII-I liegt somit ein verkürzter Akkord vor.

Die Fortschreitung des kleinen Durseptakkordes seinen Ton über sich in den Dreyklang « ${ }^{37}$ erklärt Kirnberger wiederum durch den suneigentlichen ‘ Charakter dieses Septakkordes. Dessen Septime sei »eigentlich die None des wahren Grundtones [...], deren Auflösung erst in dem folgenden Accord geschiehet. ${ }^{38}$ Dies verwundert insofern, als die eingeschränkte Möglichkeit der stufenweisen `Grundbaß -Progression bei Kirnberger/Schulz durchaus besteht. ${ }^{39}$ Problematisch gestaltet sich die möglicherweise deswegen ausgelassene Übertragung in eine Molltonart, da der analoge »eigentliche» Akkord ein übermäßiger Septakkord wäre, der als »wesentliche« Septakkordform nicht genannt wird: 40

30 Kirnberger/Schulz 1773, 20.

31 Ebd., 19.

32 Kirnberger 1771, 90.

33 Ebd., 38.

34 Ebd.

35 Ebd., 47.

36 Vgl. ebd., 66.

37 Ebd., 62.

38 Ebd.

39 Vgl. Kirnberger/Schulz 1773, 51. Hier ordnen Kirnberger/Schulz den Sekundstieg im `Grundbaß dem Prototyp I-II-V-I zu, obschon eine Terzsubstruktion VI-II-V-I ebenfalls möglich wäre, sofern die erste Stufe nicht per Quintfall erreicht wird.

40 Der Moll-Trugschluss wird lediglich als Beispiel ohne Grundbass integriert; vgl. Kirnberger 1771, 98. Zum übermäßigen Dreiklang bemerkt Kirnberger: „Wenn man den Linien nach Terzen, Quinten 


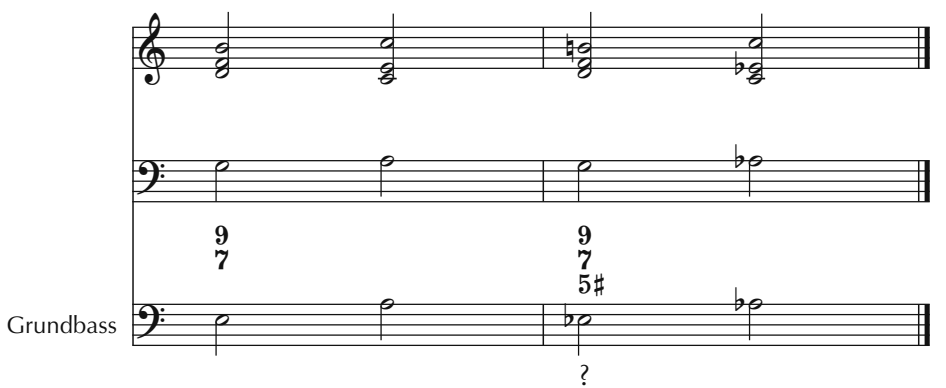

Beispiel 7: Übertragung von Kirnbergers Trugschlusserklärung (links) in eine Molltonart (rechts)

Um das Fortschreiten des Fundamentalbasses in Sekundschritten zu limitieren, beschränken sich Kirnberger/Schulz in nahezu allen Fällen auf die Folge I-II(7) $-V^{(7)}-I$, also den Einstieg in die zweite oder dritte prototypische Kadenz über den Akkord der I. Auch die Kadenz I-IV $-\mathrm{V}$ in einer Dur-Tonart involviere den Septnonakkord der II mit verzögert aufgelöster zzufälliger` Nondissonanz. ${ }^{41}$ Zur Dreiklangsfolge I-IV-V-I bemerkt der Autor der Wahren Grundsätze hingegen:

Dem Anschein nach sind dies lauter Dreyklänge, und der Baß des Exempels scheint der Grundbaß zu seyn, der von dem zweyten zum dritten Accord um eine Secunde fortschreitet: Aber der zweyte Accord verträgt die Sexte neben sich, und ist daher kein Grundaccord, sondern ein Quintsextenaccord, der die Unterterz des Baßtones zum Grundton hat, wodurch die Fortschreitung in der Secunde aufgehoben wird. ${ }^{42}$

Dies erreicht gegenüber den verzögerten Vorhalten von Septnonakkorden insofern eine neue Qualität der `Grundbaß«-Behandlung, als hier ein in jeder Hinsicht konsonanter Dreiklang zum dissonierenden Septakkord erklärt wird. ${ }^{43}$ Die Begründung dieses Sachverhaltes richtet sich offensichtlich nach der Möglichkeit der Ergänzung der IV um ihre Sexte bei konstanter tonaler Funktion (»verträgt die Sexte neben sich ${ }^{44}$ ), wiederum im Sinne des swesentlichen Septakkordes der zweiten oder dritten prototypischen Position. Da auf diese Weise die oben erwähnte, unproblematische Progression I- II - V-I entstünde, nimmt es wunder, dass der `Grundbaß an der fraglichen Stelle zusätzlich in IV-II aufgeteilt wird. Die zur weiteren Demonstration der >Grundbaß -Bestimmung

u.d.g. durch die Anzahl der Grade hervorbringen will, so verfällt man in Irrthümer, woraus alle möglichen Disharmonien entstehen können. Wer könnte z.E. folgende Dreyklänge ertragen?« $(1771,39)$

41 Ebd., 66.

42 Kirnberger/Schulz 1773, 51.

43 Der oben genannte konsonante Charakter des verminderten Dreiklangs ist auch im damaligen Kontext als Ausnahmelehre Kirnbergers zu werten; vgl. etwa Marpurg 1776, 290.

44 Es handelt sich in der Terminologie Rameaus wiederum um einen mitgehörten Ton ('sous-entendus). 
den Wahren Grundsätzen angefügte „Nacherinnerung «" ${ }^{45}$ kommt dessen ungeachtet in der identischen Folge I-IV-V-I zu dem `Grundbaß I-IV-V-I. Hier ist das System des swahren Grundbasses` also entweder mehrdeutig oder aber der fraglichen Autorschaft der Wahren Grundsätze geschuldet. Zumindest der aufgeteilte `Grundbaß`sowie die Darstellung konsonanter Dreiklänge als verkürzte ıwesentliche`Septakkorde lassen sich meiner Übersicht zufolge nur in den Wahren Grundsätzen nachweisen.

\section{Simon Sechter}

Im Kompositionssystem Simon Sechters emanzipieren sich virtuelle Grundtöne von der Funktion eines Erklärungsmodells für bestimmte Fortschreitungen, indem sie als vverschwiegene Fundamente für alle Sekundfortschreitungen des Fundamentalbasses herangezogen werden. Da ihr Gebrauch somit regelhaft vereinheitlicht wird, entstehen Probleme weniger in konkurrierenden Deutungen gleicher satztechnischer Phänomene, als vielmehr in der Konsistenz ihrer Verbindung zum realen Tonsatz.

Wie Rameau und Kirnberger führt Sechter alle Harmonien auf konsonante Dreiklänge und dissonante Septakkorde zurück. ${ }^{46}$ Dabei gilt ihm der verminderte Dreiklang als dissonant und dessen Quintton als vorbereitungsbedürftig. ${ }^{47}$ Neben allen Dissonanzen muss nach Sechter auch der Quint- oder Grundton eines konsonanten Akkordes ${ }^{48}$ im Sinne eines sharmonischen Bindemittels` vorbereitet werden. Auf diese Weise leistet er dem Gebot der >Fundamentalfortschreitungs in Terzen oder Quinten Vorschub. Während Rameau den Vorrang dieser Verbindungen aus der Partialtonreihe ableitet und Kirnberger dieselbe Priorität empirisch begründet, gemahnt die Sechter'sche Darstellung an einen Zirkelschluss: Das harmonische Band der Quintenvorbereitung einerseits und die Terz- und Quintfortschreitungen des Fundamentalbasses andererseits begleiten einander ohne die Fundierung des einen durch das andere. Die »natürliche Beziehung « ${ }^{49}$ der genannten Schritte wird also nicht hergeleitet, sondern gleichsam als eine Grundlage des Systems gesetzt. Das nachfolgende Beispiel zeigt, wie bei den empfohlenen Progressionen entweder Grund- oder Quintton der Folgeharmonie vorbereitet werden.

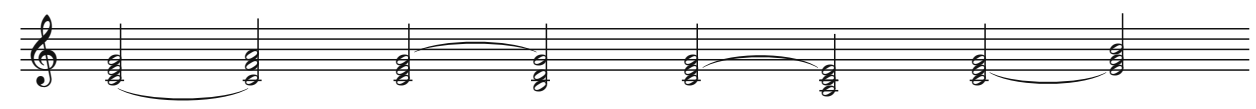

Beispiel 8: Vorbereitung des Grund- oder Quinttons bei den durch Sechter erlaubten Quintund Terzfortschreitungen

45 Kirnberger/Schulz 1773, 104.

46 Sechter 1853, 4.

47 Ebd., 12. Zeleny verweist auf den nicht konsequenten Gebrauch des verminderten Dreiklangs durch Sechter in dessen Generalbassschule (1938, 431).

48 Sechter 1853, 14: „Bei der Quint muss entweder sie selbst oder der Ton, wogegen sie eine Quint macht, vorbereitet sein und eines davon stufenweise weiter gehen«.

49 Ebd., 15. 
Sekundfortschreitungen zwischen Grundtönen müssen nach Sechter `Schlussfällen nachgebildet sein, indem ein zusätzlicher Basston »entweder wirklich gemacht oder hinein gedacht ${ }^{50}$ wird. Der bei Kirnberger/Schulz sporadisch auftretende aufgeteilte Fundamentschritt ${ }^{51}$ wird so als sverschwiegenes Fundament zu einem Grundsatz der Sechter'schen Lehre. Die Behandlung des betreffenden Quinttones (also des Septimtones über dem gedachten Fundament) als Dissonanz könnte den sverschwiegenen` Wechsel als notwendig legitimieren. Robert W. Wason weist jedoch in diesem Zusammenhang anhand von Sechters eigenen Beispielen auf die begrenzte Vereinbarkeit von Dissonanzbehandlung und Parallelenverbot hin. ${ }^{52}$ Ein offensichtliches Beispiel für ein solches unausweichliches Stimmführungsproblem bietet die Folge IV-V in einer Molltonart. Soll hier ein Stimmzug aufwärts über die Terztöne der beiden Akkorde geführt werden, so müssen diese im Sinne der melodischen Mollskala hochalteriert werden. Nach der Theorie des iverschwiegenen Fundamentes` wird zusätzlich das Fundament der zweiten Stufe eingeschoben. Dadurch erhält der Quintton der IV die Funktion eines Septimtones und müsste demzufolge abwärts aufgelöst werden. Dies wiederum würde zwangsweise zu einer Leittonverdopplung führen. Somit »bleibt kein Mittel übrig, als die Sept aufwärts gehen zu lassen, welches darum geschehen kann, da man das Fundament nicht hört. ${ }^{53}$ Anstatt von einer Ausnahmeerscheinung auszugehen oder das Auslassen des vermeintlichen Septimtones generell vorzuschreiben, verzichtet Sechter hier streng genommen auf alle Stimmführungskonsequenzen des iverschwiegenen Fundamentes` und somit auf dessen Fundierung durch den realen Satz. Eine weitere Bekräftigung erfährt dieses Vorgehen im dritten Teil der Grundsätze: „Wenn überdies das erste Fundament verschwiegen wird, wodurch eine unhörbare Sept frei angewendet kann, so ergeben sich noch folgende Gestaltungen dieser Harmoniefolge [C-(A)-D] für den strengen Satz. « ${ }^{54}$

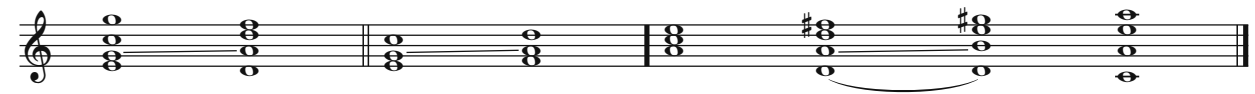

Beispiel 9: Simon Sechter, Die Grundsätze der musikalischen Komposition, durch sstumme Fundamente entstehende 'Stimmführungsfehler ${ }^{55}$

Der halbverminderte Septakkord wird im duralen Kontext analog zu Kirnbergers Theorie je nach Fortschreitung als ınatürlicher $\mathrm{VII}^{7}$ oder als künstlicher $\mathrm{V}^{7 / 9}$ ohne Dominantgrundton beschrieben. Da Sechter Septnonakkorden als verzögerten Vorhalten zur

50 Ebd., 18.

51 Zeleny sieht den aufgeteilten Fundamentschritt ebenfalls in der Kirnberger'schen Ableitung des »Trugschlusses« (1938, 431f.). Tatsächlich aber erklärt Kirnberger diesen durch einen genuinen Septnonakkord (1771, 66f.).

52 Wason 1985, $41 \mathrm{f}$.

53 Sechter 1853, 76.

54 Sechter 1854, 106. Wason führt ein ähnliches Beispiel an $(1985,42)$.

55 Sechter 1854, 106 und Sechter 1853, 76. 
Oktave eine »uneigentliche Selbständigkeit» ${ }^{56}$ zumisst, können hier die Fundamente ohne verschwiegenen Wechsel ausgelassen werden. Das betrifft ebenfalls den vollverminderten Septakkord als - modern gesprochen - sverkürzten $V^{7 / 9>}$. Sowohl aus dieser Grundtonbestimmung, als auch aus den oben besprochenen Zwischenfundamenten wird deutlich, dass die Sechter'sche 'Fundamentalstimme « wiederum nur anhand des unmittelbaren harmonischen Umfeldes zu erklären ist.

Im Gegensatz zum swahren Grundbaß bei Kirnberger/Schulz werden bei Sechter zu einer Akkordfolge häufig mehrere alternative Fundamente aufgeführt. ${ }^{57}$ Damit wird der `Fundamentalbass` zu einem eher pädagogisch intendierten Kriterium von Progressionen mit nur eingeschränktem Bezug zur realen Akkordgestalt. Er erhält dabei die Doppelfunktion, einerseits die Erzeugung von Harmoniefolgen zu unterstützen und andererseits Sätze auf ihre `Natürlichkeit hin zu untersuchen.

\section{Vergleich}

Die Geschichte der vom Basston abweichenden harmonischen Grundtonbestimmung in der theoretischen Literatur war bis zu Rameau von der Umschichtung/Oktavversetzung von Akkordtönen geprägt. ${ }^{58} \mathrm{Zu}$ diesem vertikal orientierten Prinzip tritt nun die Abhängigkeit der Fundamente von horizontalen Progressionen. Besonders prägend wirkt sich dabei Rameaus Idee eines aus der Natur des Klanges abgeleiteten Grundprinzips aus, da dieses zu einer Erklärung aller harmonischen Bewegungen als Imitate oder Derivate der scadence parfaite ‘ führt. In Anbetracht der zahlreichen diesbezüglichen Herleitungsmodelle Rameaus entstehen Widersprüche besonders durch die unscharfe Trennung von spekulativer Begründung und pragmatischer Anwendung in der Analyse.

Die Systeme Kirnbergers und Sechters können vor diesem Hintergrund als Versuche verstanden werden, die widersprüchliche bzw. unübersichtliche Fundamentalbasslehre zu vereinheitlichen. Kirnberger entwickelt durch seine Theorie der ızufälligen und ınotwendigen • Dissonanzen implizit die zweigliedrige Kadenzbildung Rameaus zu einem mehrgliedrigen Fortschreitungsprototyp und integriert ebenfalls die charakteristischen Dissonanzen als snotwendige septimtöne. In Widersprüche verwickelt er sich jedoch bei der Bestimmung des swahren Grundbaß durch Duldung von sekundschrittigen Progressionen und deren gleichzeitiger Erklärung durch interpolierte Bässe.

Sechter entwirft ein logisch relativ geschlossenes Erklärungssystem, das durch eine axiomatische Setzung stumme Fundamente als grundlegende Prämissen einbezieht. Es stellt sich jedoch die Frage, inwieweit der Fundamentalbass mit den für die Behandlung einzelner Akkordbestandteile als Kon- oder Dissonanzen verantwortlichen Grundtönen korreliert.

Die historische Etablierung der Methode kann anhand zweier Begründungsstrategien dargestellt werden. Stumme Fundamente entstehen erstens durch die Verknüpfung eines Akkordtyps mit einer prototypischen Verbindung und zweitens durch Umdeutungen zur

56 Sechter 1853, 30.

57 Z.B. ebd., 83, 139 und Sechter 1854, 111.

58 Vgl. Lester 1994, 53-56 und 96ff. 
Umgehung eines Sekundschrittes im Fundamentalbass. Als entscheidende Idee wirkt dabei der dem sprincipe، geschuldete Vorrang von terz- oder quintweisen Fundamentalbassbewegungen, der jedoch für Kirnberger und Sechter insofern irrelevant erscheint, als sie nicht wie Rameau rationalistisch, sondern vielmehr historisch/empirisch (Kirnberger) oder systematisch/axiomatisch (Sechter) argumentieren.

Rameau liefert in diesem Prozess lose kombinierte Muster als Einzelerklärungen, die erst von anderen Autoren sukzessiv zu stummen Fundamenten umgeformt wurden. Zu diesen Mustern gehören der ambivalente Grundton (`double emploi`), die Schwächung des Bestimmungsprinzips der Terzenschichtung ('supposition`, Grundtöne spar emprunt`) sowie schließlich der zwiefache Charakter der Fundamentalbassstimme als bloße Folge von Grundtönen und Regulierungsstimme des musikalischen Satzes. Letzterer Aspekt entwickelte sich in logischer Hinsicht besonders problematisch, da schon bei Rameau einerseits Erkenntnisse für den harmonischen Satz aus der `basse fondamentale sewonnen werden, während diese Stimme andererseits umgeformt wird, um diese Erkenntnisse zu bestätigen.

\section{Literatur}

Christensen, Thomas (1993), Rameau and Musical Thought in the Enlightenment, Cambridge: Cambridge University Press.

Dahlhaus, Carl (1989), Die Musiktheorie im 18. und 19. Jahrhundert - Zweiter Teil: Deutschland, hg. von Ruth E. Müller, Darmstadt: Wissenschaftliche Buchgesellschaft.

Kirnberger, Johann Philipp, (1771), Die Kunst des reinen Satzes in der Musik, Berlin: Voss, Reprint Kassel: Bärenreiter 2004.

— (1781), Grundsätze des Generalbasses, Berlin: Hummel, Reprint Hildesheim: Olms 1974.

Kirnberger, Johann Philipp / Johann Abraham Peter Schulz (1773), Die wahren Grundsätze zum Gebrauch der Harmonie, Berlin und Königsberg: Decker und Hartung, Reprint Hildesheim: Olms 1970.

Lester, Joel (1992), Compositional Theory in the Eighteenth Century, Cambridge: Cambridge University Press.

Martin, Nathan John (2012), »Rameau's Changing Views on Supposition and Suspension ", Journal of Music Theory 56/2, 121-167.

Marpurg, Friedrich Wilhelm (1776), Versuch über die musikalische Temperatur, Breslau: Korn.

Rameau, Jean-Philippe (1722), Traité de I'harmonie réduite à ses principes naturels, Paris: Ballard, Reprint Genève: Slatkine 1986.

- (1760), Code de musique pratique, Paris: L'imprimerie Royale, zit. nach: Ders., Complete theoretical writings, Bd. IV, hg. von Erwin Reuben Jacobi, Dallas: American Institute of Musicology 1969. 
Sechter, Simon (1853), Die Grundsätze der musikalischen Komposition. Erste Abtheilung, Leipzig: Breitkopf und Härtel.

(1854), Die Grundsätze der musikalischen Komposition. Dritte Abtheilung, Leipzig: Breitkopf und Härtel.

Wason, Robert W. (1985), Viennese Harmonic Theory from Albrechtsberger to Schenker and Schoenberg, Rochester, NY, Woodbridge, Suffolk: University of Rochester Press.

Zeleny, Walter (1938), Die historischen Grundlagen des Theoriesystems von Simon Sechter (= Wiener Veröffentlichungen zur Musikwissenschaft 10), Diss. Universität Wien, Erstdruck hg. von Othmar Wessely, Tutzing: Schneider 1979. 